\title{
The Vulnerable Plaque: the Real Villain in Acute Coronary Syndromes
}

\author{
Michael Liang ${ }^{1}$, Aniket Puri ${ }^{1,2}$ and Gerard Devlin ${ }^{1, *}$ \\ ${ }^{1}$ Department of Cardiology, Waikato Hospital, New Zealand \\ ${ }^{2}$ Department of Cardiology, CSM Medical University (Former King George Medical University), Lucknow, India
}

\begin{abstract}
The term "vulnerable plaque" refers to a vascular lesion that is prone to rupture and may result in lifethreatening events which include myocardial infarction. It consists of thin-cap fibroatheroma and a large lipid core which is highly thrombogenic. Acute coronary syndromes often result from rupture of vulnerable plaques which frequently are only moderately stenosed and not visible by conventional angiography. Several invasive and non-invasive strategies have been developed to assess the burden of vulnerable plaques. Intravascular ultrasound provides a two-dimensional crosssectional image of the arterial wall and can help assess the plaque burden and composition. Optical coherent tomography offers superior resolution over intravascular ultrasound. High-resolution magnetic resonance imaging provides non-invasive imaging for visualizing fibrous cap thickness and rupture in plaques. In addition, it may be of value in assessing the effects of treatments, such as lipid-lowering therapy. Technical issues however limit its clinical applicability. The role of multi-slice computed tomography, a well established screening tool for coronary artery disease, remains to be determined. Fractional flow reserve (FFR) may provide physiological functional assessment of plaque vulnerability; however, its role in the management of vulnerable plaque requires further studies. Treatment of the vulnerable patient may involve systemic therapy which currently include statins, ACE inhibitors, beta-blockers, aspirin, and calcium-channel blockers and in the future local therapeutic options such as drug-eluting stents or photodynamic therapy.
\end{abstract}

Keywords: Vulnerable plaque, coronary artery disease.

\section{INTRODUCTION}

Atherosclerosis is a systemic disease of the vessel wall. The main components of the atherosclerotic plaque are connective tissue extracellular matrix, including collagen, proteoglycans, and fibronectin elastic fibers; cholesterol, cholesteryl esters, and phospholipids; and cells such as monocyte-derived macrophages, $\mathrm{T}$ lymphocytes, and smooth muscle cells. Varying proportions of these components occur in different plaques, giving rise to a spectrum of lesions [1, 2]. The term vulnerable plaque is used to describe a plaque at high risk of disruption leading to thrombosis. Vulnerable plaques tend to have a thin fibrous cap and a large lipid core which is highly thrombogenic [3, 4]. Acute coronary syndromes often result from rupture of these angiograpically mildly stenosed lesions. The caps are often thinnest at the shoulder region, where macrophages and mast cells accumulate. In addition there is a large lipid core rich in cholesterol, also containing cholesteryl esters which facilitate plaque softening. A negative relation exists between temperature and core stiffness. If temperature increases, as in inflammation, the core becomes softer. A soft core may be more vulnerable to rupture as it may not be able to withstand the imposed circumferential stress, which is then redistributed to the fibrous cap [5-7]. It is well

\footnotetext{
*Address correspondence to this author at the Clinical Unit Leader, Cardiology, Cardiothoracic Surgery and Vascular Surgery Unit. Waikato Hospital, Pembroke \& Selwyn Sts, Hamilton 3240, New Zealand; Tel: 0064-7-839-8899; Fax: 0064-7-839-8799; Email: gerard.devlin@waikatodhb.health.nz
}

recognised that a haemodynamically non-significant coronary plaque can rupture and produce a cardiac event long before it produces significant lumen narrowing and symptoms of angina.

Attempts have been made to predict individual risk of development of severe atheroscelerosis from populationbased risk factors studies. Traditional cardiovascular risk factors including age, gender, body mass index, diabetes, hypertension, smoking, lipid profile and family history of premature coronary artery disease have been established over the last 60 years from population-based studies such as the Framingham heart study and the Prospective Cardiovascular Munster (PROCAM) study [8, 9]. However, a recent retrospective study of the utility of the National Cholesterol Education Program (NCEP) III guidelines in a group of young adults (age <or $=55$ years for men and <or $=65$ years for women), reported that only, $25 \%$ of the patients who had recently experienced acute myocardial infarction met the criteria for pharmacological therapy [10]. Consequently many additional tools and scoring systems exist to help identify patients at risk not only of developing cardiovascular disease but acute coronary syndromes. This manuscript reviews current concepts of the vulnerable plaque and patient and discuss diagnosis and current and future treatment options.

\section{The Concept of Vulnerable Plaque and Patient}

Vulnerable plaques may result in vulnerable high-risk patients. This concept has recently been discussed by Naghavi et al. in a consensus document which concluded the 
following [11-13]. Firstly rupture-prone plaques are not the only vulnerable plaques. All types of atherosclerotic plaques with a high likelihood of thrombotic complications and rapid progression should be considered as vulnerable plaques. Secondly vulnerable plaques are not the only causal factors for the development of acute coronary syndromes. Vulnerable blood (prone to thrombosis) and vulnerable myocardium (prone to fatal arrhythmia) play equally important roles. The investigators propose criteria for defining vulnerable plaque, based on the examination of culprit plaques, and markers of vulnerability. Major criteria suggested are active inflammation, presence of thin cap with large lipid core, endothelial denudation with superficial platelet aggregation, fissured plaque or presence of high grade coronary stenosis. Minor criteria are superficial calcified nodule, presence of plaque hemorrhage, endothelial dysfunction, or positive remodeling of the artery. Markers of vulnerability at the plaque level include morphology such as cap thickness, the size of lipid core size, the colour of the plaque, collagen content, lipid content, stiffness, calcification and flow pattern throughout the coronary artery. The presence of features which suggest the presence of a vulnerable plaque identifies an individual at higher risk of an acute coronary syndrome presentation - hence the term 'vulnerable patient'.

\section{The Vulnerable Patient and Identification by Serum Markers}

The term "vulnerable patient" may be more appropriate to clinicians. This concept of a vulnerable patient, combined with the wide acceptance of atherosclerosis as a chronic inflammatory disease, has resulted in a series of new approaches to risk stratification in cardiovascular disease detection of serum inflammatory markers, blood thrombogenicity and matrix degrading capacity that correlate with coronary artery disease [14]. The high-sensitive C-reactive protein (hsCRP) is one of the most established serum markers and is currently available in routine clinical practice. CRP is an acute phase protein with a stable baseline concentration which increases during systemic inflammation. Over 40 clinical studies had investigated the potential association of inflammation with cardiovascular disease and the perhaps more importantly the ability of hsCRP levels to predict future cardiovascular events $[15,16]$. The use and interpretation of hsCRP is however limited by the fact that it is an acute phase protein which is elevated in diseases or injuries causing inflammation and tissue damage which unfortunately translates into a high false positive rate in the ability of hsCRP to predict future cardiovascular events. A metaanalysis of 22 population-based prospective studies however reported an odds ratio of 1.6 with CRP in predicting a cardiovascular event after correction for several established cardiovascular risk factors [17]. In addition, the JUPITER investigators have reported that rosuvastatin is equally as effective in men and women in reducing major adverse cardiac events in a population without hyperlipidemia but considered at increased cardiovascular risk due to an elevated hs-CRP [18]. The largest and most comprehensive metaanalysis to date looking at CRP levels and cardiovascular risk, included patients from JUPITER and examined the individual records of 160309 people from 54 long-term prospective studies with $48 \%$ of the subjects female. The re- searchers concluded that whilst CRP is unlikely to be a causal factor for cardiovascular disease it may help identify patients at increased risk who can benefit from early intervention [19].

Other inflammatory markers such as interleukin (IL)-6, IL-8, IL-3, Macrophage colony-stimulating factor (M-CSF) and soluble CD40 ligand have reported similar odds ratios to hsCRP in the ability to improve diagnosis over conventional risk factors. Again, these inflammatory markers have low positive predictive value due to their low specificity. In addition to the inflammatory markers, many novel serum markers such as fibrinogen, myeloid-related protein $8 / 14$, adiponectin, brain natriuretic peptide and matrix metalloproteinase-9 (MMP-9) have been investigated and appear to add little to the Framingham risk score [14, 20].

\section{DIAGNOSIS OF VULNERABLE PLAQUE}

Despite significant advances in imaging this remains problematic. Most current techniques identify luminal diameter or stenosis, wall thickness, and plaque volume but remain unable to identify high-risk plaques. High-resolution, multicontrast magnetic resonance imaging (MRI) appears to currently hold the most promise, particularly of noninvasive imaging of high-risk vulnerable or rupture prone plaques. The diagnostic modalities currently available to the cardiologist and their usefulness and limitations are listed in Table 1. These can essentially be divided into non-invasive and invasive techniques.

\section{NON-INVASIVE MODALITIES}

The enthusiasm for use of non-invasive modalities to help identify vulnerable plaque was inspired by the first SHAPE (Screening for Heart Attack Prevention and Education) guideline. The investigators observed that most heart attacks and strokes occur in people who are not classified at high risk by traditional cardiovascular risk factors and therefore, proposed non-invasive screening for asymptomatic men (45-75years old) and women (55-75 years old) [21]. The currently available non-invasive modality used to attempt to diagnose the patient with vulnerable plaque are electronbeam computed tomography (EBCT), multislice CT (MSCT), CT coronary angiography (CTA), magnetic resonant imagine (MRI) and nuclear techniques such as nuclear scintigraphy, positron-emission tomography (PET) and PET-CT.

The traditional EBCT is a technique for imaging coronary artery calcium which utilizes a faster rate of image acquisition than conventional computed tomography. However, it has no proven correlation with plaque vulnerability as vulnerable plaque often lacks calcium. MSCT and CTA, on the other hand, shows good correlation with coronary angiography in assessing stenosis severity, with a sensitivity between $93-95 \%$, specificity of $85-90 \%$ [20, 22]. In addition, MSCT are able to distinguish fibrous-rich from lipid-rich plaques and providing more information on plaque morphology [23]. In terms of radiation exposure, MSCT is regarded as a moderate to high. A recent publication by SmithBindman et al., suggests the risk of this should not be underestimated particularly in women. In this retrospective cross - 
Table 1. Information Provided by Various Imaging Modalities to Identify the Vulnerable Plaque

\begin{tabular}{|c|c|c|c|c|c|c|c|c|c|}
\hline & Summary Feature Detected & $\begin{array}{l}\text { Lumen } \\
\text { Stenosis }\end{array}$ & $\begin{array}{l}\text { Vessel } \\
\text { Wall }\end{array}$ & $\begin{array}{l}\text { Lipid } \\
\text { Content }\end{array}$ & $\begin{array}{l}\text { Fibrous } \\
\text { Tissue }\end{array}$ & Calcium & Thrombus & Inflam'n & $\begin{array}{l}\text { Predict } \\
\text { Events }\end{array}$ \\
\hline \multicolumn{10}{|l|}{ Invasive Modalities } \\
\hline Angiography & Degree of stenosis & + & - & - & - & $+/-$ & $+/-$ & - & $+/-$ \\
\hline $\begin{array}{l}\text { IVUS (Elastography, } \\
\text { palpography and } \\
\text { virtual histology) }\end{array}$ & $\begin{array}{l}\text { Plaque volume, vessel dimensions, } \\
\text { (plaque deformability and } \\
\text { composition) }\end{array}$ & + & + & $+/-$ & + & + & $+/-$ & - & $+/-$ \\
\hline Angioscopy & Plaque Color & - & - & + & + & - & + & - & $+/-$ \\
\hline OCT & Plaque architecture and cap thickness & + & + & + & + & + & + & - & $?$ \\
\hline Thermography & PlaqueTemperature & - & - & $?$ & $?$ & $?$ & $?$ & + & $+/-$ \\
\hline Raman/NIR & Plaque chemical constituents & - & - & + & + & + & - & - & ? \\
\hline \multicolumn{10}{|c|}{ Non-Invasive Modalidies } \\
\hline Nuclear Scintigraphy & Plaque constituents & - & - & + & - & - & + & - & ? \\
\hline EBCT & Predominantly calcium & + & - & - & - & + & - & - & $+/-$ \\
\hline MSCT & Vessel characteristics & + & + & + & + & + & - & - & $?$ \\
\hline MRI & Plaque volume & + & + & + & + & + & - & - & $?$ \\
\hline
\end{tabular}

sectional study the investigators estimated life-time attributable cancer risk associated with imaging and concluded one in 270 women who underwent a CT coronary angiogram at age 40 will eventually develop cancer as compared to one in 600 men [24]. With improved technology and particulary increased acquisition speeds the clinical usefulness of MSCT in identifying vulnerable plaque warrants further investigation.

High-resolution magnetic resonance imagine (MRI) has emerged as a potential noninvasive in vivo imaging modality for atherosclerotic plaque characterization. MRI differentiates plaque components on the basis of biophysical and biochemical parameters which include chemical composition and concentration, water content, physical state, molecular motion, or diffusion. In addition, MRI also provides details of surrounding tissue in one session. One main advantage of MRI over MSCT is the ability to perform imaging without ionizing radiation. In addition it can be repeated sequentially over time. High-resolution MRI is an excellent tool for visualizing fibrous cap thickness and rupture in coronary plaques $[25,26]$. At present, MRI provides not only a method of noninvasively visualizing plaque and discriminating its components but also a way of accurately assessing the effects of treatments, such as lipid-lowering therapy, and timing the activity of clots to determine when they become inactive. These techniques, whilst promising remain experimental.

Nuclear imaging which includes PET and PET-CT have a higher detection sensitivity than MRI, however, remain compromised by relatively lower resolution which impairs imaging of small vessels such as coronary arteries. Many radiotracers have been developed on the basis of molecules and cells involved in atherogenesis. Of note, 18F-Fluoro-2DeoxyGlucose (18F-FDG) which normally accumulates more quickly in tumour cells has also been observed to exhibit early uptake in plaque macrophages, and as such may have a role in non-invasive detection of highly inflamed vulnerable plaque $[27,28]$. To date, however, no single radiotracer appears ideally suited to image atherosclerosis and provide the prognostic and clinical indicators necessary for medical and surgical interventions.

\section{INVASIVE IMAGING MODALITIES}

Invasive imaging modalities include coronary angiography, intravascular ultrasound (IVUS), and optical coherent tomography (OCT). Other modalities such as thermography, angioscopy, Raman spectrography and near-infrared spectrography are currently investigational tools and are not discussed in this manuscript.

\section{Coronary Angiography}

It is well established that, although an individual severe coronary stenosis may become acutely occluded, this is a more frequent occurrence in less obstructive plaques [29]. Indeed, in approximately $70 \%$ of patients presenting with myocardial infarction, the lesions leading to occlusion have been reported as $<50 \%$ stenotic $[1,29]$. In the early phases of atherosclerosis the luminal size is not affected by plaque growth due to expansion of the external elastic membrane, so-called "positive remodeling". As the plaque grows and approaches the lumen, negative remodeling occurs. Positive remodeling is also seen in an acute myocardial infarction at the site of plaque rupture, whereas negative remodeling and 
smaller plaque areas may be associated with stable angina [30]. This phenomenon of remodeling makes angiography a poor technique with which to assess the true atherosclerotic disease burden as the shadows of the lumen seen on angiography provide only indirect and incomplete information concerning the extent of the atherosclerosis process in the arterial wall.

\section{Intravascular Ultrasound}

Intravascular ultrasound (IVUS) provides a twodimensional cross-sectional image of the arterial wall and can accurately assess the plaque burden [31]. On the basis of echogenicity, plaques can be differentiated into three categories as follows: (i) highly echoreflective area corresponding to calcified tissue; (ii) hyperechoic areas representing fibrosis or (iii) hypoechoic regions corresponding to thrombus or lipid-rich tissue [32]. Histopathologic studies mostly report low sensitivity and specificity for IVUS in detecting lipidrich lesions, and axial resolution remains too low for measuring cap thickness. Efforts have focused on novel methods to analyze the integrated backscatter of the signal to improve plaque characterization. A commercially available system has been developed using this analysis that assigns plaque to the categories of fibrotic, fibrofatty, calcific, and necrotic core and has been termed "virtual histology" [33]. Another addition to conventional IVUS examination is the use of elastography. This concept is based on the principle that tissue components can differ in hardness as a result of their different histopathologic composition and are expected to be compressed differently if a defined pressure is applied. The technique can discriminate between soft and hard material and can assess the mechanical properties of the vessel wall. Hard calcifications will be compressed less than soft lipids [34]. The strain images are constructed using the relative local displacements and hard and soft regions can be identified using this technique. With intravascular imaging a derivative of elastography called palpography is used. Elastography has the potential to identify plaque vulnerability as the detected areas of increased radial strain represent regions of high circumferential stress, a feature of plaque vulnerability [34]. However, a major problem in advancing intravascular elastography to cardiac in vivo applications is the acquisition of data in a pulsating artery located in a contracting heart.

\section{Optical Coherence Tomography}

Optical coherence tomography (OCT) is analogous to IVUS, but measures the intensity of reflected infrared light rather than acoustic waves [33, 35]. Studies have shown OCT is capable of differentiating lipid tissue and furthermore, the thickness of the fibrous cap overlying an atheroma can be well demarcated [33, 35]. However despite widespread use in areas such as ophthalmology there are still many limitations of OCT for in vivo intravascular imaging including the reduction of image quality through blood or large volumes of tissue, the relative slow data acquisition rate, and the multiple scattering. The information acquired by OCT and IVUS are complimentary as OCT can produce high resolution images to help assesss fibrous cap thickness and macrophage infiltrations of intact coronary atheroscle- rotic plaques while IVUS provides details of penetration and tissue characterization of the lesions.

\section{PHYSIOLOGICAL ASSESSMENT}

Fractional flow reserve (FFR) is increasingly used to identify physiological important lesions which angiographically appear non-flow limiting. It is defined as a ratio of the pressure distal to a stenosis in relation to the pressure proximal to the stenosis under maximum hyperaemia, normally induced by either intra-coronary or peripheral adenosine infusion. A FFR of 0.75 means that a given stenosis causes a $25 \%$ drop in blood flow pressure which is considered as a significant stenosis. The DEFER investigators reported deferral of percutaneous coronary intervention in intermediate lesions with an FFR of $>0.75$ is safe with a low risk of acute coronary syndrome presentations; $<1 \%$ after 5 years of follow-up [36, 37]. The Fractional Flow Reserve Versus Angiography for Multivessel Evaluation (FAME) trial further demonstrated that the use of FFR in patients with multiple vessel coronary who are undergoing percutaneous coronary intervention with drug-eluting stents significantly reduces the mortality, nonfatal myocardial infarction and revascularization at 1 year compared with angiographically-guided group [38]. In addition, Versteeg et al. demonstrated that the monocyte toll-like receptors 2 and 4, which are related to plaque vulnerability, were significantly higher in patients with FFR $<0.75$ than in patients with an FFR of $>0.80$. This suggests that plaque vulnerability may be preceded by ischaemia [39]. To date, there is no clinical trial to date using FFR to detect vulnerable plaque per se, therefore, the role of FFR in the detection of vulnerable plaques and management of vulnerable patients requires further evaluation.

\section{MANAGEMENT}

Innovations in both medical therapy and novel interventional techniques have significantly decreased the morbidity and mortality associated with coronary atherosclerosis. However, detection of vulnerable patients remains elusive; as for many individuals, sudden coronary death is the first presentation with cardiac disease. For primary prevention, the emphasis is on plasma markers and noninvasive testing to identify patients at risk. For secondary prevention, interest has focused on vulnerable patients with vulnerable plaques they may possess that may be identified and treated at the time of cardiac catheterization. It is plausible that many agents already proven to prevent coronary events by reducing plaque vulnerability.

\section{Systemic Therapy for Vulnerable Plaque}

The pharmacological therapies of proven benefit in the management of vulnerable plaque -related coronary events are statins, ACE inhibitors, beta-blockers, aspirin, and calcium-channel blockers [40]. There is no doubt that intense lowering of LDL, which may be achieved by statin therapy, can prevent clinical events initiated by vulnerable plaques. The ASTEROID study reported that a marked reduction in LDL was associated with an $11.1 \%$ decrease in coronary plaque volume as measured by IVUS. It is plausible that intense LDL lowering was responsible for this beneficial decrease in plaque volume and stabilization of plaques [41]. 
Angiotensin-converting enzyme (ACE) inhibitors may improve plaque stability by inhibiting the endothelial dysfunction and oxygen-free radical production caused by angiotensin. In addition, they may also decrease macrophage activity and inhibit smooth muscle cell lipoxygenase activity. it is possible that the benefit of ACE inhibitors is in part due to up-regulation of type III collagen synthesis, or an antiatherogenic effect as suggested by the HOPE study subgroup. These investigators demonstrated a significant reduction in the rate of carotid intimal medial thickening with ramipril [42, 43]. Calcium antagonists may also stabilise plaques by interfering with lipid oxidation and reducing foam cell formation which accompanies a significant increase in transmembrane calcium transport seen in acute coronary syndromes implying an anti-atherogenic role [42]. Antithrombotic agents are of established benefit in the management of acute coronary syndromes, in addition, the European Society for Vascular Surgery guidelines recommended the use of antithrombotic agents for both symptomatic and asymptomatic carotid stenosis patients in whom vulnerable plaques have a role in the disease process [44-46]. These act mainly by reducing intravascular haemostasis, but may also have a direct passivating effect on the vascular wall. Aspirin has antiplatelet and anti-inflammatory properties and has demonstrated benefits both as a stand-alone treatment in acute coronary syndrome, or in combination with heparin and clopidogrel. In addition, as platelets promote the accumulation of inflammatory cells, the antiplatelet aggregation activity of Gp IIb/IIIa inhibitors can reduce plaque macrophage burden [42].

Many other agents may have a role in the management of vulnerable plaques but require further clinical validation include antioxidants, folic acid, antibiotics, angiotensinreceptor blockers, omega-3 fatty acids, cyclooxygenase- 2 inhibitors, influenza vaccine, metalloproteinase inhibitors, peroxisome proliferator-activated receptor antagonists, Lipoprotein-associated phospholipase A2, darapladib, AGI1067 and cholesterol ester transfer protein inhibitors [40, 42, 47].

\section{LOCAL THERAPY FOR VULNERABLE PLAQUE}

Although systemic therapy is the likely preferred option for most individuals, the currently available treatment options do not provide adequate protection, particularly for the very-high-risk patient. The opportunity exists in these individuals to consider delivery of local preventive therapy in addition to systemic therapy at the time of revascularization procedures.

Local delivery of therapy to the vulnerable plaque may be considered particulary if regions at increased risk of a clinical cardiac event can be identified. Promising local treatment options under investigation include stents with or without drugs and photodynamic therapy.

Most lesions responsible for acute coronary syndromes occur in the proximal part of the major coronary arteries as these are regions of shear stress with increased numbers of thin-cap fibroatheromas. Stenting, a logical option for issues relating to increased mechanical stress is an effective treat- ment of established coronary disease. Furthermore improvements in stent technology continue and are likely to reduce the risks associated with stenting and thereby shift the risk-to benefit ratio in favour of stenting less severe lesions. Drug-eluting stents have been evaluated in an atherosclerotic rabbit model as a possible treatment for vulnerable plaques. Placement of a stent over lesions in the aorta reduced lipid core size and induced formation of an additional fibrous cap over the lipid pool. Although these experimental results are encouraging, the benefits of stenting angiographically mild to moderate lesions on the rationale they contain vulnerable plaques must be confirmed in randomized trials. Bioabsorbable stents, which may reduce or eliminate the long-term risk of stent thrombosis, may be preferable for prophylactic stenting of nonstenotic vulnerable plaques. The challenges of identification of the vulnerable plaque therefore remains pivotal to the development of local therapeutic solution.

Photodynamic therapy has been proposed as a method to stabilize a specific plaque or a region of an artery by selective ablation of macrophages or other targeted cells. The photosensitizing agent motexafin lutetium has been tested in atherosclerotic rabbits. After photoactivation via an intraaortic catheter, a marked reduction in the number of macrophages and a small decrease in atheroma burden without damage to normal tissue was observed. Photodynamic therapy has also been demonstrated to reduce neointimal growth without suppressing re-endothelialization of a stent in a porcine model $[48,49]$. The same agent has been administered to patients undergoing coronary stenting in a safety study and was found to be well tolerated. These early results suggest that photodynamic therapy may eventually have a role in the local or regional treatment of vulnerable plaques.

\section{CONCLUSION}

Understanding the concept of vulnerable plaques and vulnerable patients is helpful in the management and prevention of cardiovascular events. Despite recent advances in non-invasive and invasive imaging, identification remains a diagnostic challenge. A possible future approach in cardiovascular risk prediction and prevention may include traditional risk factor assessment, biochemical markers and affordable non-invasive imaging. Patients identified at high risk of plaque rupture-related events may be offered further assessment and treatment with high-resolution imaging and local therapies such as stenting or photodynamic therapy. The early detection of vulnerable patients will hopefully lead to early clinical treatment or interventions to prevent a life-threatening cardiovascular event.

\section{CONFLICT OF INTERESTS}

Nil

\section{ABBREVIATIONS}

\begin{tabular}{|c|c|c|}
\hline $\mathrm{ACE}$ & $=$ & Angiotensin Converting Enzynme \\
\hline CTA & $=$ & $\begin{array}{l}\text { Computed Tomographic } \\
\text { Angiography }\end{array}$ \\
\hline EBCT & $=$ & Electron-Beam Computed Tomography \\
\hline FFR & $=$ & Fractional Flow Reserve \\
\hline
\end{tabular}




$\begin{array}{lll}\text { hSCRP } & = & \text { high-sensitive C-Reactive Protein } \\ \mathrm{IL} & = & \text { InterLeukin } \\ \text { IVUS } & = & \text { IntraVascular UltrouSound } \\ \text { M-CSF } & = & \text { Macrophage Colony-Stimulating Factor } \\ \text { MMP } & = & \text { Matrix MetalloProteinase } \\ \text { MRI } & = & \text { Magnetic Resonance Imaging } \\ \text { MSCT } & = & \text { Multi-Slice Computed Tomography } \\ \text { OCT } & = & \text { Optical Coherent Tomography } \\ \text { PET } & = & \text { Positron-Emission Tomography } \\ \text { PET-CT } & = & \text { Positron-Emission Tomography Com- }\end{array}$

\section{REFERENCE}

[1] Fuster V, Fayad ZA, Badimon JJ. Acute coronary syndromes: biology. Lancet 1999; 353(Suppl 2): SII5-9.

[2] Libby P. Molecular bases of the acute coronary syndromes. Circulation 1995; 91: 2844-50.

[3] Falk E, Shah PK, Fuster V. Coronary plaque disruption. Circulation 1995; 92: 657-71.

[4] Fuster V, Lewis A. Conner Memorial Lecture. Mechanisms leading to myocardial infarction: insights from studies of vascular biology. Circulation 1994; 90: 2126-46.

[5] Loree HM, Tobias BJ, Gibson LJ, Kamm RD, Small DM, Lee RT. Mechanical properties of model atherosclerotic lesion lipid pools. Arterioscler Thromb 1994; 14: 230-4.

[6] Small DM. George Lyman Duff memorial lecture. Progression and regression of atherosclerotic lesions. Insights from lipid physical biochemistry. Arteriosclerosis 1988; 8: 103-29.

[7] Lundberg B. Chemical composition and physical state of lipid deposits in atherosclerosis. Atherosclerosis 1985; 56: 93-110.

[8] Kannel WB, Dawber TR, Kagan A, Revotskie N, Stokes J, 3rd. Factors of risk in the development of coronary heart disease--six year follow-up experience. The Framingham Study. Ann Intern Med 1961; 55: 33-50.

[9] Assmann G, Cullen P, Schulte H. The Munster Heart Study (PROCAM). Results of follow-up at 8 years. Eur Heart J 1998; 19: A2-11.

[10] Akosah KO, Schaper A, Cogbill C, Schoenfeld P. Preventing myocardial infarction in the young adult in the first place: how do the National Cholesterol Education Panel III guidelines perform? J Am Coll Cardiol 2003 7; 41: 1475-9.

[11] Naghavi M, Libby P, Falk E, et al. From vulnerable plaque to vulnerable patient: a call for new definitions and risk assessment strategies: Part I. Circulation 2003; 108: 1664-72.

[12] Naghavi M, Libby P, Falk E, et al. From vulnerable plaque to vulnerable patient: a call for new definitions and risk assessment strategies. Part II. Circulation 2003; 108: 1772-8.

[13] Naghavi M, Falk E, Hecht HS, et al. From vulnerable plaque to vulnerable patient--Part III: Executive summary of the screening for heart attack prevention and education (SHAPE) task force report. Am J Cardiol 2006; 98: 2H-15H.

[14] Packard RR, Libby P. Inflammation in atherosclerosis: from vascular biology to biomarker discovery and risk prediction. Clin Chem 2008; 54: 24-38.

[15] Danesh J, Erqou S, Walker M, et al. The Emerging Risk Factors Collaboration: analysis of individual data on lipid, inflammatory and other markers in over 1.1 million participants in 104 prospective studies of cardiovascular diseases. Eur J Epidemiol 2007; 22: 839-69.

[16] Casas JP, Shah T, Hingorani AD, Danesh J, Pepys MB. C-reactive protein and coronary heart disease: a critical review. J Intern Med 2008; 264: 295-314.

[17] Danesh J, Wheeler JG, Hirschfield GM, et al. C-reactive protein and other circulating markers of inflammation in the prediction of coronary heart disease. N Engl J Med 2004; 350: 1387-97.

[18] Ridker PM, Danielson E, Fonseca FA, et al. Rosuvastatin to prevent vascular events in men and women with elevated Creactive protein. N Engl J Med 2008; 359: 2195-207.
[19] Kaptoge S, Di Angelantonio E, Lowe G, et al. C-reactive protein concentration and risk of coronary heart disease, stroke, and mortality: an individual participant meta-analysis. Lancet 2010; 375: $132-40$.

[20] Eijgelaar WJ, Heeneman S, Daemen MJ. The vulnerable patient: refocusing on the plaque? Thromb Haemost 2009; 102: 231-9.

[21] Naghavi M, Falk E, Hecht HS, Shah PK. The first SHAPE (Screening for Heart Attack Prevention and Education) guideline. Crit Pathw Cardiol 2006; 5: 187-90.

[22] Ropers D, Rixe J, Anders K, et al. Usefulness of multidetector row spiral computed tomography with 64- x $0.6-\mathrm{mm}$ collimation and 330-ms rotation for the noninvasive detection of significant coronary artery stenoses. Am J Cardiol 2006; 97: 343-8.

[23] Schroeder S, Kuettner A, Leitritz M, et al. Reliability of differentiating human coronary plaque morphology using contrastenhanced multislice spiral computed tomography: a comparison with histology. J Comput Assist Tomogr 2004; 28: 449-54.

[24] Smith-Bindman R, Lipson J, Marcus R, et al. Radiation dose associated with common computed tomography examinations and the associated lifetime attributable risk of cancer. Arch Intern Med 2009; 169: 2078-86.

[25] Helft G, Worthley SG, Fuster V, et al. Progression and regression of atherosclerotic lesions: monitoring with serial noninvasive magnetic resonance imaging. Circulation 2002; 105: 993-8.

[26] Yuan C, Zhang SX, Polissar NL, et al. Identification of fibrous cap rupture with magnetic resonance imaging is highly associated with recent transient ischemic attack or stroke. Circulation 2002; 105: 181-5.

[27] Ogawa M, Ishino S, Mukai T, et al. (18)F-FDG accumulation in atherosclerotic plaques: immunohistochemical and PET imaging study. J Nucl Med 2004; 45: 1245-50.

[28] Chen W, Bural GG, Torigian DA, Rader DJ, Alavi A. Emerging role of FDG-PET/CT in assessing atherosclerosis in large arteries. Eur J Nucl Med Mol Imaging 2009; 36: 144-51.

[29] Alderman EL, Corley SD, Fisher LD, et al. Five-year angiographic follow-up of factors associated with progression of coronary artery disease in the Coronary Artery Surgery Study (CASS). CASS Participating Investigators and Staff. J Am Coll Cardiol 1993; 22: 1141-54.

[30] Burke AP, Kolodgie FD, Farb A, Weber D, Virmani R. Morphological predictors of arterial remodeling in coronary atherosclerosis. Circulation 2002; 105: 297-303.

[31] Losordo DW, Rosenfield K, Kaufman J, Pieczek A, Isner JM. Focal compensatory enlargement of human arteries in response to progressive atherosclerosis. In vivo documentation using intravascular ultrasound. Circulation 1994; 89: 2570-7.

[32] Nissen SE, Yock P. Intravascular ultrasound: novel pathophysiological insights and current clinical applications. Circulation 2001; 103: 604-16.

[33] Garcia-Garcia HM, Gonzalo N, Regar E, Serruys PW. Virtual histology and optical coherence tomography: from research to a broad clinical application. Heart 2009; 95: 1362-74.

[34] de Korte CL, Pasterkamp G, van der Steen AF, Woutman HA, Bom N. Characterization of plaque components with intravascular ultrasound elastography in human femoral and coronary arteries in vitro. Circulation 2000; 102: 617-23.

[35] Brezinski ME, Tearney GJ, Bouma BE, et al. Optical coherence tomography for optical biopsy. Properties and demonstration of vascular pathology. Circulation 1996; 93: 1206-13.

[36] Sharif F, Murphy RT. Current status of vulnerable plaque detection. Catheter Cardiovasc Interv 2010 1; 75: 135-44.

[37] Pijls NH, van Schaardenburgh P, Manoharan G, et al. Percutaneous coronary intervention of functionally nonsignificant stenosis: 5year follow-up of the DEFER Study. J Am Coll Cardiol 2007; 49: 2105-11.

[38] Tonino PA, De Bruyne B, Pijls NH, et al. Fractional flow reserve versus angiography for guiding percutaneous coronary intervention. N Engl J Med 2009; 360: 213-24.

[39] Versteeg D, Hoefer IE, Schoneveld AH, et al. Monocyte toll-like receptor 2 and 4 responses and expression following percutaneous coronary intervention: association with lesion stenosis and fractional flow reserve. Heart 2008; 94: 770-6.

[40] Ambrose JA, D'Agate DJ. Classification of systemic therapies for potential stabilization of the vulnerable plaque to prevent acute myocardial infarction. Am J Cardiol 2005; 95: 379-82. 
[41] Nissen SE, Nicholls SJ, Sipahi I, et al. Effect of very high-intensity statin therapy on regression of coronary atherosclerosis: the ASTEROID trial. JAMA 2006; 295: 1556-65.

[42] Spratt JC, Camenzind E. Plaque stabilisation by systemic and local drug administration. Heart 2004; 90: 1392-4.

[43] Yusuf S, Sleight P, Pogue J, Bosch J, Davies R, Dagenais G. Effects of an angiotensin-converting-enzyme inhibitor, ramipril, on cardiovascular events in high-risk patients. The Heart Outcomes Prevention Evaluation Study Investigators. N Engl J Med 2000; 342: 145-53.

[44] Liapis CD, Bell PF, Mikhailidis DP, et al. ESVS Guidelines: section $\mathrm{B}$ - diagnosis and investigation of patients with carotid stenosis. Curr Vasc Pharmacol 2010; 8: 682-91.

[45] Liapis CD, Bell PF, Mikhailidis DP, et al. ESVS Guidelines: section A - prevention in patients with carotid artery stenosis. Curr Vasc Pharmacol 2010; 8: 673-81.
[46] Liapis CD, Bell PR, Mikhailidis D, et al. ESVS guidelines. Invasive treatment for carotid stenosis: indications, techniques. Eur J Vasc Endovasc Surg 2009; 37: 1-19.

[47] Serruys PW, Garcia-Garcia HM, Buszman P, et al. Effects of the direct lipoprotein-associated phospholipase $\mathrm{A}(2)$ inhibitor darapladib on human coronary atherosclerotic plaque. Circulation 2008; 118: 1172-82.

[48] Kereiakes DJ, Szyniszewski AM, Wahr D, et al. Phase I drug and light dose-escalation trial of motexafin lutetium and far red light activation (phototherapy) in subjects with coronary artery disease undergoing percutaneous coronary intervention and stent deployment: procedural and long-term results. Circulation 2003; 108: 1310-5.

[49] Waksman R, Leitch IM, Roessler J, et al. Intracoronary photodynamic therapy reduces neointimal growth without suppressing re-endothelialisation in a porcine model. Heart 2006; 92: 1138-44.

(C) Liang et al.; Licensee Bentham Open.

This is an open access article licensed under the terms of the Creative Commons Attribution Non-Commercial License (http://creativecommons.org/licenses/ by-nc/3.0/) which permits unrestricted, non-commercial use, distribution and reproduction in any medium, provided the work is properly cited. 